\title{
SMOOTH STEADY SOLUTIONS OF THE PLANAR VLASOV-POISSON SYSTEM WITH A MAGNETIC OBSTACLE
}

\author{
BY
}

\author{
JACK SCHAEFFER
}

Department of Mathematics Sciences, Carnegie Mellon University, Pittsburgh, Pennsylvania 15213

\begin{abstract}
The solar wind interacting with a magnetized obstacle is modeled with the Vlasov equation. The domain considered is a disk in the plane. Inflowing boundary conditions are given for the particle density. A magnetic field is prescribed, and the electric field is computed self consistently with potential zero on the boundary. Taking the boundary condition for the particle density to be sufficiently small, it is shown that there is a natural smooth steady solution. The speed of the inflowing plasma and the magnetic field are not size restricted.
\end{abstract}

1. Introduction. Let $R>0, D=\left\{x \in \mathbb{R}^{2}:|x|<R\right\}$, and $\partial D=\left\{x \in \mathbb{R}^{2}:|x|=R\right\}$. Consider the problem

$$
\left\{\begin{array}{l}
v \cdot \nabla_{x} f+\left(E_{1}+v_{2} B\right) \partial_{v_{1}} f+\left(E_{2}-v_{1} B\right) \partial_{v_{2}} f=0 \text { on } D \times \mathbb{R}^{2} \\
f \text { given if } x \in \partial D \text { and } x \cdot v<0 \\
\rho=\int f d v \\
\Delta U=-4 \pi \rho \text { in } D \\
U=0 \text { on } \partial D \\
E=-\nabla U
\end{array}\right.
$$

where $B$ is a given function of $x$. Realistic modeling would require including an additional species of particles (with opposite charge), but this does not affect the methods used here, so it is omitted. It will be assumed that $B$ has support contained in $D$. The boundary condition that $U=0$ on $\partial D$ says physically that the boundary is a perfect conductor.

Received January 15, 2013.

2010 Mathematics Subject Classification. Primary 35L60, 35Q83, 82C22, 82D10.

E-mail address: js5m@andrew.cmu.edu 
This is spurious, but in order to work on a bounded domain, some boundary condition must be given. This one gives a physically meaningful problem.

Let

$$
\begin{aligned}
& S_{+}=\left\{(x, v) \in \partial D \times \mathbb{R}^{2}: x \cdot v>0\right\}, \\
& S_{-}=\left\{(x, v) \in \partial D \times \mathbb{R}^{2}: x \cdot v<0\right\}
\end{aligned}
$$

and

$$
S_{0}=\left\{(x, v) \in \partial D \times \mathbb{R}^{2}: x \cdot v=0\right\} .
$$

Theorem 1.1. Let $B \in C^{1}\left(\mathbb{R}^{2}\right)$ and assume there is $C_{1} \in(0, R)$ such that

$$
B(x) \neq 0 \Rightarrow|x|<C_{1} .
$$

Let $F: S_{-} \cup S_{0} \rightarrow[0, \infty)$ be $C^{1}$ and assume there are $C_{2}, C_{3} \in(0, \infty)$ such that

$$
F(x, v) \neq 0 \Rightarrow C_{2}>|v|>C_{3} \text { and } v \cdot \frac{(-x)}{R}>C_{3} .
$$

We also assume (1.5), which is stated below. Then there exists $C>0$ such that for every $\varepsilon \in(0, C)$ there exists $f \in C^{1}\left(\bar{D} \times \mathbb{R}^{2}\right)$ and $U \in C^{2}(\bar{D})$ that satisfy (1.1) where the boundary condition for $f$ is

$$
f=\varepsilon F \text { on } S_{-} .
$$

\section{Comments}

1. In general, we do not expect uniqueness for the above problem. If there are characteristics which never intersect the boundary, then the value of $f$ on these is not determined by (1.4); hence we expect uniqueness to fail. For the solution constructed in Theorem 1.1, $f$ is zero along any characteristic that never intersects the boundary. Thus, all charge comes from upstream by way of condition (1.4).

2. Restricting $\varepsilon$ limits the amount of charge the plasma carries. In the limit as $\varepsilon \rightarrow 0^{+}, E \rightarrow 0$ and the motion of the particles is determined by

$$
\left\{\begin{aligned}
\frac{d X}{d s} & =V \\
\frac{d V}{d s} & =\left(V_{2} B(X),-V_{1} B(X)\right) .
\end{aligned}\right.
$$

Condition (1.5) requires that when $\varepsilon=0$ the particles exit the domain within a bounded time.

3. $B$ and the $v$ support of $F$ are not size restricted. Thus, the inflowing plasma may move rapidly and encounter a large magnetic obstacle and hence change direction rapidly. Study of this problem was motivated by interest in the bow shock formed when the solar wind encounters the earth's magnetic field (see [14] for example). 
4. The well-known theorems that ensure the existence of smooth solutions ([8, [9]) apply to the time dependent problem. The bounds they obtain increase without bound as $t$ increases and hence cannot be used for the time independent case. Many papers on the steady problem (e.g. [1, 4]) concern solutions of the form

$$
f(x, v)=G\left(\frac{1}{2}|v|^{2}+U(x)\right) .
$$

However, this would require

$$
f(x, v)=G\left(\frac{1}{2}|v|^{2}\right) \text { on } \partial D \times \mathbb{R}^{2},
$$

which conflicts with (1.4).

For excellent surveys of the mathematics of Vlasov equations see [3] and [10. In the landmark papers [9] and [8] it is shown that solutions of the Poisson-Vlasov system in three space dimensions which start smooth remain smooth for all time. We mention [11, 12, [13] as other attempts to model a steady solar wind. Other papers that consider boundary value problems for the Vlasov equation are [5, 6], 7]. In particular, it was pointed out in [6] that regularity can be lost near characteristics that intersect the boundary in a tangential manner. Much effort is made in this work to ensure the solution is zero in a neighborhood of any such tangential characteristic. One factor in this is assumption (1.3). Another is the restriction of $\varepsilon$ which in turn restricts the size of $E$. Note, also, that $B=0$ near $\partial D$ by (1.2).

The letter $C$ will denote a generic positive constant which changes from line to line and may depend on $B, F, R$ but not on $\varepsilon$ or $n$. When a specific constant is chosen it will be given a subscript. For example, $C_{1}, C_{2}, C_{3}$ introduced in Theorem 1.1 will have the same values throughout the paper. The norm

$$
\|E\|_{\infty}=\sup \{|E(x)|: x \in D\}
$$

will be used. Also $\bar{D}=D \cup(\partial D)$ and

$$
\left(v_{1}, v_{2}\right) \wedge B=\left(v_{2} B,-v_{1} B\right) .
$$

Consider the case $\varepsilon=0$ first. For $(x, v) \in \mathbb{R}^{2} \times \mathbb{R}^{2}$ define $\left(X^{0}(s, x, v), V^{0}(s, x, v)\right)$ by

$$
\begin{cases}\frac{d X^{0}}{d s}=V^{0} & X^{0}(0, x, v)=x \\ \frac{d V^{0}}{d s}=V^{0} \wedge B\left(X^{0}\right) & V^{0}(0, x, v)=v .\end{cases}
$$

For $(x, v) \in S_{-}$define

$$
\omega^{0}(x, v)=\sup \{t>0: X(s, x, v) \in D \forall s \in(0, t)\}
$$

and assume there exists $T^{0}>0$ such that

$$
\omega^{0}(x, v) \leq T^{0}
$$

for all $(x, v) \in S_{-}$with $F(x, v) \neq 0$. 
Lemma 1.1. There exists $C_{4}>0$ such that $X^{0}\left(\omega^{0}(x, v), x, v\right) \in \partial D$ and

$$
V^{0}\left(\omega^{0}(x, v), x, v\right) \cdot n \geq C_{4},
$$

where $n=R^{-1} X^{0}\left(\omega^{0}(x, v), x, v\right)$, for all $(x, v) \in S_{-}$with $F(x, v) \neq 0$.

Proof. For brevity we drop the dependence on $(x, v)$. It follows by a standard continuity argument (and since $\omega^{0}$ is finite) that $X^{0}\left(\omega^{0}\right) \in \partial D$. Note, also, that for all $s \in\left(0, \omega^{0}\right)$,

$$
\left|V^{0}(s)\right|=|v| \text {. }
$$

Consider the case that $B\left(X^{0}(s)\right) \neq 0$ for some $s \in\left(0, \omega^{0}\right)$. Define

$$
t_{1}=\sup \left\{t \in\left(s, \omega^{0}\right):\left|X^{0}\right| \leq C_{1} \text { on }(s, t)\right\} .
$$

Then $\left|X^{0}\right|>C_{1}$ on $\left(t_{1}, \omega^{0}\right)$ so $B\left(X^{0}\right)=0$ and $V^{0}$ is constant on $\left[t_{1}, \omega^{0}\right]$. Using some geometry it follows that

$$
\sin \theta \leq C_{1} / R
$$

where $\theta$ is the angle between $V\left(\omega^{0}\right)$ and $n$. Hence

$$
V^{0}\left(\tau^{0}\right) \cdot n=|v| \cos \theta \geq C_{3} \cos \left(\sin ^{-1}\left(\frac{C_{1}}{R}\right)\right)=C .
$$

Now consider the case that $B\left(X^{0}(s)\right)=0$ for all $s \in\left(0, \omega^{0}\right)$. Then $V^{0}(s)=v$ for all $s \in\left[0, \omega^{0}\right]$ and

completing the proof.

$$
V^{0}\left(\tau^{0}\right) \cdot n=v \cdot\left(\frac{-x}{R}\right)>C_{3}
$$

2. The linear Vlasov equation. In this section, we consider $E \in C^{1}(\bar{D})$ given. A solution of the linear Vlasov equation will be defined that satisfies

$$
f=\varepsilon F \quad \text { on } \quad S_{-} \cup S_{0} .
$$

The goal of this section is to show that there is a constant $C>0$ such that for $\|E\|_{\infty}<C$ we have $f \in C^{1}\left(\bar{D} \times \mathbb{R}^{2}\right)$ and

$$
\|f\|_{\infty}+\|\nabla f\|_{\infty} \leq C \varepsilon
$$

Consider $(x, v) \in\left(D \times \mathbb{R}^{2}\right) \cup S_{-} \cup S_{+}$. Define $(X(s, x, v), V(s, x, v))$ by

$$
\left\{\begin{array}{l}
\frac{d X}{d s}=V \quad X(0, x, v)=x \\
\frac{d V}{d s}=E(X)+V \wedge B(X) \quad V(0, x, v)=v .
\end{array}\right.
$$

Next we define $f$. On $S_{-} \cup S_{0}$ define

$$
f=\varepsilon F .
$$

Consider $(x, v) \in\left(D \times \mathbb{R}^{2}\right) \cup S_{+}$. Define

$$
\alpha(x, v)=\inf \{t<0: X(s, x, v) \in D \quad \forall s \in(t, 0)\}
$$

and then

$$
f(x, v)=\varepsilon F(X(\alpha(x, v), x, v), V(\alpha(x, v), x, v))
$$


if $\alpha(x, v)$ is finite and

$$
f(x, v)=0
$$

otherwise.

When $f(x, v) \neq 0$ we may bound $\alpha(x, v)$ by using $T^{0}$ :

Lemma 2.1. There exists $C_{5}>0$ such that for $\|E\|_{\infty}<C_{5}$ the following holds: Consider $(x, v) \in S_{-}$with $F(x, v) \neq 0$ and define

$$
\omega(x, v)=\sup \{t>0: X(s, x, v) \in D \quad \forall s \in(0, t)\} .
$$

Then

$$
\omega(x, v) \leq 2 T^{0}
$$

and

where

$$
V(\omega(x, v), x, v) \cdot n \geq \frac{1}{2} C_{4}
$$

$$
n=R^{-1} X(\omega(x, v), x, v) .
$$

Proof. Dropping the dependence on $(x, v)$ we have

$$
\begin{aligned}
& \left|\frac{d V}{d s}-\frac{d V^{0}}{d s}\right|=\left|E(X)+V \wedge B(X)-V^{0} \wedge B\left(X^{0}\right)\right| \\
\leq & \|E\|_{\infty}+\left|V-V^{0}\right||B(X)|+\left|V^{0}\right|\left|B(X)-B\left(X^{0}\right)\right| \\
\leq & \|E\|_{\infty}+C\left(\left|X-X^{0}\right|+\left|V-V^{0}\right|\right) .
\end{aligned}
$$

Hence, for $s \in\left(0, \min \left(\omega, 2 T^{0}\right)\right)$ we have

$$
\left|X(s)-X^{0}(s)\right|+\left|V(s)-V^{0}(s)\right| \leq 2 T^{0}\|E\|_{\infty}+C \int_{0}^{s}\left(\left|X-X^{0}\right|+\left|V-V^{0}\right|\right) d u,
$$

and Gronwall's inequality yields

$$
\begin{aligned}
\left|X(s)-X^{0}(s)\right|+\left|V(s)-V^{0}(s)\right| & \leq 2 T\|E\|_{\infty} e^{C s} \\
& \leq 2 T^{0}\|E\|_{\infty} e^{C 2 T^{0}}=C\|E\|_{\infty} .
\end{aligned}
$$

Now we sketch the rest of the proof. Let

$$
\mathcal{E}^{0}=V^{0} \cdot\left(\left|X^{0}\right|^{-1} X^{0}\right) \text { and } \mathcal{E}=V \cdot\left(|X|^{-1} X\right) .
$$

For illustration consider the case when $\omega<\omega^{0}$ (and hence $\omega<T^{0}$ ). Since we may restrict $\|E\|_{\infty}$, (2.6) allows us to restrict $\left|X(\omega)-X^{0}(\omega)\right|+\left|V(\omega)-V^{0}(\omega)\right|$, and hence

$$
R-\left|X^{0}(\omega)\right|=|X(\omega)|-\left|X^{0}(\omega)\right|
$$

may be made as small as desired. As in the proof of Lemma 1.1 we may obtain

$$
\mathcal{E}^{0} \geq C
$$

on $\left[\omega, \omega^{0}\right]$. It follows that $\omega-\omega^{0}$ may be made arbitrarily small and that

$$
\mathcal{E}(\omega) \geq \mathcal{E}^{0}\left(\omega^{0}\right)-\left|\mathcal{E}^{0}(\omega)-\mathcal{E}^{0}\left(\omega^{0}\right)\right|-\left|\mathcal{E}^{0}(\omega)-\mathcal{E}(\omega)\right| \geq C .
$$

The case when $\omega \geq \omega^{0}$ may be handled similarly, so the proof is complete. 


\section{Comment}

It follows from Lemma 2.1 that if $(x, v) \in\left(D \times \mathbb{R}^{2}\right) \cup S_{+}$and $f(x, v) \neq 0$, then

$$
\alpha(x, v) \geq-2 T^{0} .
$$

Lemma 2.2. There is $C_{6} \in\left(0, C_{5}\right]$ such that if $\|E\|_{\infty}<C_{6}$, then $f \in C^{1}\left(\bar{D} \times \mathbb{R}^{2}\right)$.

Proof. We'll take

$$
\|E\|_{\infty}<C_{6}=\min \left(C_{5}, \frac{1}{4} C_{3}\left(2 T_{0}\right)^{-1},\left(\frac{1}{4} C_{3}\right)^{2} R^{-1}\right) .
$$

Consider $(x, v) \in\left(D \times \mathbb{R}^{2}\right) \cup S_{+}$. If $f(x, v) \neq 0$, then $\alpha(x, v) \geq-2 T^{0}$ so

$$
|V(\alpha(x, v), x, v)-v| \leq\|E\|_{\infty}|\alpha|<\frac{1}{4} C_{3}
$$

But

$$
f(x, v)=\varepsilon F(X(\alpha(x, v), x, v), V(\alpha(x, v), x, v)) \neq 0,
$$

So

$$
|V(\alpha(x, v), x, v)| \geq C_{3}
$$

and

So

$$
|v|>\frac{3}{4} C_{3}
$$

$$
|v| \leq \frac{3}{4} C_{3} \Rightarrow f(x, v)=0 .
$$

Suppose $\alpha(x, v) \geq-2 T^{0}$ and

$$
X(\alpha(x, v), x, v) \cdot V(\alpha(x, v), x, v)=0 .
$$

We claim that there is a neighborhood of $(x, v)$ on which

$$
f(y, w)=0 .
$$

To show this, note that (writing $X(\alpha)=X(\alpha(x, v), x, v)$, etc.) $|X(\alpha)|^{2}=R^{2}$,

$$
\left.\frac{d}{d s}|X(s)|^{2}\right|_{s=\alpha}=0
$$

and $|X(s)|^{2} \leq R^{2}$ for all $s \in[\alpha, 0]$, so

$$
\begin{aligned}
0 & \geq\left.\frac{d^{2}}{d s^{2}}|X(s)|^{2}\right|_{s=\alpha}=2|V(\alpha)|^{2}+2 X(\alpha) \cdot E(X(\alpha)) \\
& \geq 2\left(|V(\alpha)|^{2}-R\|E\|_{\infty}\right)
\end{aligned}
$$

and

Hence

$$
|V(\alpha)| \leq \sqrt{R\|E\|_{\infty}} \leq \frac{1}{4} C_{3}
$$

$$
|v| \leq|V(\alpha)|+\|E\|_{\infty}|\alpha| \leq \frac{1}{4} C_{3}+\|E\|_{\infty} 2 T^{0}<\frac{1}{2} C_{3} .
$$

Now (2.8) follows from (2.7). 
Suppose $\alpha(x, v) \in\left[-\infty,-2 T^{0}\right)$. Then there is a neighborhood of $(x, v)$ on which $\alpha(y, w) \in\left[-\infty,-2 T^{0}\right)$ and hence

$$
f(y, w)=0 .
$$

Suppose $\alpha(x, v) \geq-2 T^{0}$ and

$$
X(\alpha(x, v), x, v) \cdot V(\alpha(x, v), x, v) \neq 0 .
$$

Then there is a neighborhood of $(x, v)$ on which

$$
\alpha(y, w) \geq-3 T^{0}
$$

and

$$
f(y, w)=\varepsilon F(X(\alpha(y, w), y, w), V(\alpha(y, w), y, w)) .
$$

On this neighborhood $f$ is the composition of $C^{1}$ functions.

Finally, for $(x, v) \in S_{0}$,

$$
f(y, w)=0
$$

on a neighborhood of $(x, v)$, and if $(x, v) \in S_{-}$there is a neighborhood of $(x, v)$ on which $f$ is the composition of $C^{1}$ functions. Thus the proof is complete.

Lemma 2.3. There exists $C_{7}>0$ such that if $\|E\|_{\infty}<C_{6}$ we have

$$
f(x, v) \neq 0 \Rightarrow|v| \leq C_{7}
$$

and

$$
\int f(x, v) d v \leq C_{7} \varepsilon .
$$

Proof. Assume $f(x, v) \neq 0$. If $(x, v) \in S_{-} \cup S_{0}$, then $f(x, v)=\varepsilon F(x, v)$ so $|v| \leq C_{2}$. Suppose $(x, v) \in\left(D \times \mathbb{R}^{2}\right) \cup S_{+}$. By (2.7) $|V(s)| \neq 0$ for all $s \in[\alpha, 0]$, so

$$
\begin{aligned}
|v| & =|V(\alpha)|+\int_{\alpha}^{0} \frac{V(s)}{|V(s)|} \cdot E(X(s)) d s \\
& \leq C_{2}+|\alpha|\|E\|_{\infty} \leq C_{2}+2 T^{0} C_{6} .
\end{aligned}
$$

Also, (2.12) follows since $\|f\|_{\infty}=\|\varepsilon F\|_{\infty}$, completing the proof.

Lemma 2.4. There exists $C_{8}>0$ such that

$$
\|\nabla f\|_{\infty} \leq C_{8} e^{C_{8}\|\nabla E\|_{\infty}} \varepsilon
$$

if $\|E\|_{\infty}<C_{6}$.

Proof. Let $(x, v) \in S_{-}$. Then $f(X(s), V(s))=\varepsilon F(x, v)$, and since $f$ is $C^{1}$ it follows that

$$
v \cdot \nabla_{x} f+(E+v \wedge B) \cdot \nabla_{v} f=0 .
$$


By using a regularization argument it follows that

$$
\begin{aligned}
\partial_{x_{i}} f(X(s), V(s))= & \varepsilon \partial_{x_{i}} F(x, v) \\
& -\int_{0}^{s}\left(\partial_{x_{i}} E(X)+V \wedge \partial_{x_{i}} B(X)\right) \cdot \nabla_{v} f(X, V) d u
\end{aligned}
$$

for $i=1,2$,

$$
\begin{aligned}
\partial_{v_{1}} f(X(s), V(s))= & \varepsilon \partial_{v_{1}} F(x, v) \\
& -\int_{0}^{s}\left(\partial_{x_{1}} f(X, V)-B(X) \partial_{v_{2}} f(X, V)\right) d u
\end{aligned}
$$

and

$$
\begin{aligned}
\partial_{v_{2}} f(X(s), V(s))= & \varepsilon \partial_{v_{2}} F(x, v) \\
& -\int_{0}^{s}\left(\partial_{x_{2}} f(X, V)+B(X) \partial_{v_{1}} f(X, V)\right) d u .
\end{aligned}
$$

Hence

$$
\begin{aligned}
\left|\nabla_{x} f(X(s), V(s))\right| \leq & \left\|\nabla_{x} F\right\|_{\infty} \varepsilon \\
& +\left(\sqrt{2}\|E\|_{\infty}+C_{7}\|\nabla B\|_{\infty}\right) \int_{0}^{s}\left|\nabla_{v} f(X, V)\right| d u
\end{aligned}
$$

and

$$
\begin{aligned}
\left|\nabla_{v} f(X(s), V(s))\right| \leq & \left\|\nabla_{v} F\right\|_{\infty} \varepsilon \\
& +\int_{0}^{s}\left(\left|\nabla_{x} f(X, V)\right|+\|B\|_{\infty}\left|\nabla_{v} f(X, V)\right|\right) d u .
\end{aligned}
$$

Hence

$$
|\nabla f(X(s), V(s))| \leq C \varepsilon+C\left(1+\|\nabla E\|_{\infty}\right) \int_{0}^{s}|\nabla f(X, V)| d u,
$$

and by Gronwall's inequality

$$
\begin{aligned}
|\nabla f(X(s), V(s))| & \leq C \varepsilon e^{C\left(1+\|\nabla E\|_{\infty}\right) s} \\
& \leq C \varepsilon e^{C\left(1+\|\nabla E\|_{\infty}\right) 2 T^{0}}=C e^{C\|\nabla E\|_{\infty} \varepsilon} .
\end{aligned}
$$

The lemma now follows.

Next define

$$
\rho=\int f d v
$$

$\widetilde{U}$ by

$$
\left\{\begin{aligned}
\Delta \tilde{U} & =-4 \pi \rho & & \text { on } \bar{D} \\
\tilde{U} & =0 & & \text { on } \partial D
\end{aligned}\right.
$$

and

$$
\tilde{E}=-\nabla \tilde{U}
$$


Lemma 2.5. There exist $C_{9}>1$ such that

$$
\|\tilde{E}\|_{\infty} \leq C_{9}\|\rho\|_{\infty}
$$

and

$$
\|\nabla \tilde{E}\|_{\infty} \leq C_{9}\left(1+\|\rho\|_{\infty}\right) \ln \left(C_{9}\left(1+\|\rho\|_{\infty}+\|\nabla \rho\|_{\infty}\right)\right) .
$$

The proof is deferred to the appendix.

3. Iteration. Define $f_{0}=0, \rho_{0}=0$, and $E_{0}=0$. For $E_{n} \in C^{1}(\bar{D})$ known with $\left\|E_{n}\right\|_{\infty}<C_{6}$, define $f_{n+1}, \rho_{n+1}$, and $E_{n+1}$ as follows. Let $E=E_{n}$. Then take $f, \rho$, and $\widetilde{E}$ as in the previous section. Now define $f_{n+1}=f, \rho_{n+1}=\rho$, and $E_{n+1}=\tilde{E}$. By Lemmas 2.5 and 2.3

$$
\left\|E_{n+1}\right\|_{\infty} \leq C_{9}\left\|\rho_{n+1}\right\|_{\infty}
$$

and

$$
\left\|\rho_{n+1}\right\|_{\infty} \leq C_{7} \varepsilon
$$

We require

$$
\varepsilon<\frac{C_{6}}{C_{9} C_{7}}
$$

so that

$$
\left\|E_{n+1}\right\|_{\infty}<C_{6}
$$

By induction it follows that $f_{n}, \rho_{n}$, and $E_{n}$ are defined and satisfy

$$
\left\|E_{n}\right\|_{\infty}<C_{6}
$$

and

$$
\left\|\rho_{n}\right\|_{\infty} \leq C_{7} \varepsilon<C_{6} / C_{9}
$$

for all $n$. Also

$$
\left\|E_{n}\right\|_{\infty}<C_{9} C_{7} \varepsilon
$$

for all $n$.

Lemma 3.1. There exist $C_{10}>0$ and $C_{11}>0$ such that if $\varepsilon<C_{10}$, then

$$
\left\|\nabla E_{n}\right\|_{\infty}<C_{11}
$$

for all $n$.

Proof. By Lemmas 2.5, 2.3, 2.4 and by (3.1) we have

$$
\begin{aligned}
\left\|\nabla E_{n+1}\right\|_{\infty} & \leq C_{9}\left(1+\left\|\rho_{n+1}\right\|_{\infty}\right) \ln \left(C_{9}\left(1+\left\|\rho_{n+1}\right\|_{\infty}+\left\|\nabla \rho_{n+1}\right\|_{\infty}\right)\right) \\
& \leq\left(C_{9}+C_{6}\right) \ln \left(C_{9}+C_{6}+C_{9}\left\|\nabla \rho_{n+1}\right\|_{\infty}\right) \\
& \leq\left(C_{9}+C_{6}\right) \ln \left(C_{9}+C_{6}+C_{9} \pi C_{7}^{2} C_{8} e^{C_{8}\left\|\nabla E_{n}\right\|_{\infty} \varepsilon}\right) .
\end{aligned}
$$

So there is $C_{12}>0$ such that

$$
\left\|\nabla E_{n+1}\right\| \leq C_{12} \ln \left(C_{12}\left(1+e^{C_{12}\left\|\nabla E_{n}\right\|_{\infty}} \varepsilon\right)\right) .
$$


Take

and

$$
\begin{aligned}
& C_{11}=C_{12} \ln \left(2 C_{12}\right), \\
& C_{10}=\min \left(C_{6} C_{7}^{-1} C_{9}^{-1}, e^{-C_{12} C_{11}}\right),
\end{aligned}
$$

If $\left\|\nabla E_{n}\right\|_{\infty}<C_{11}$, then

$$
\varepsilon<C_{10}
$$

$$
\begin{aligned}
\left\|\nabla E_{n+1}\right\|_{\infty} & \leq C_{12} \ln \left(C_{12}\left(1+e^{C_{12} C_{11}} \varepsilon\right)\right) \\
& <C_{12} \ln \left(C_{12}(1+1)\right)=C_{11} .
\end{aligned}
$$

The lemma now follows by induction.

Before addressing the convergence of this iteration, a preliminary lemma is needed.

Lemma 3.2. Assume $E \in C^{1}(\bar{D})$ and $\|E\|_{\infty}<C_{6}$. For any $\delta>0$ there is $r \in\left[C_{1}, R\right)$ such that if $|x| \in[r, R)$ and

$$
v \cdot \frac{(-x)}{|x|} \geq \delta
$$

then

$$
\alpha(x, v) \geq-\frac{R-|x|}{\frac{1}{2} \delta}
$$

and

for all $s \in[\alpha(x, v), 0]$.

$$
V(s, x, v) \cdot \frac{(-X(s, x, v))}{|X(s, x, v)|} \geq \frac{1}{2} \delta
$$

Proof. Let $\alpha_{\delta}=\max \left(\alpha, \frac{-\delta}{2 C_{6}}\right)$. For $s \in\left[\alpha_{\delta}, 0\right]$,

$$
\begin{aligned}
V(s) \cdot \frac{(-X(s))}{|X(s)|}= & v \cdot \frac{(-x)}{|x|} \\
& +\int_{s}^{0}\left(E(X) \cdot \frac{X}{|X|}+\frac{|X|^{2}|V|^{2}-(X \cdot V)^{2}}{|X|^{3}}\right) d u \\
\geq & \delta-\int_{s}^{0} C_{6} d u=\delta+C_{6} s \geq \frac{1}{2} \delta
\end{aligned}
$$

and

Take

$$
|X(s)|=|x|+\int_{s}^{0} V \cdot \frac{(-X)}{|X|} d u \geq|x|-\frac{1}{2} \delta s .
$$

$$
r=\max \left(C_{1}, R-\frac{\delta^{2}}{8 C_{6}}\right) .
$$

If $\alpha \leq \frac{-\delta}{2 C_{6}}$, then $\alpha_{\delta}=\frac{-\delta}{2 C_{6}}$ and

$$
R \geq\left|X\left(\alpha_{\delta}\right)\right| \geq|x|-\frac{1}{2} \delta \alpha_{\delta} \geq r+\frac{\delta^{2}}{4 C_{6}} \geq R+\frac{\delta^{2}}{8 C_{6}},
$$


a contradiction. Hence $\alpha>\frac{-\delta}{2 C_{6}}$, and (3.4) follows from (3.5). Also by (3.6) we have

$$
R=|X(\alpha)| \geq|x|-\frac{1}{2} \delta \alpha
$$

and (3.3) follows, completing the proof.

Define $\left(X_{n+1}(s, x, v), V_{n+1}(s, x, v)\right)$ by

$$
\begin{cases}\frac{d X_{n+1}}{d s}=V_{n+1} & X_{n+1}(0, x, v)=x \\ \frac{d V_{n+1}}{d s}=E_{n}\left(X_{n+1}\right)+V_{n+1} \wedge B\left(X_{n+1}\right) & V_{n+1}(0, x, v)=v\end{cases}
$$

and

$$
\alpha_{n}(x, v)=\inf \{t<0: X(s, x, v) \in D \quad \forall s \in(t, 0)\},
$$

as in (2.1) and (2.3).

Now consider $(x, v) \in\left(D \times \mathbb{R}^{2}\right) \cup S_{+}$with either $f_{n}(x, v) \neq 0$ or $f_{n+1}(x, v) \neq 0$. We'll consider the case $f_{n}(x, v) \neq 0$; the other case may be handled similarly. Then

$$
\begin{aligned}
\alpha_{n}(x, v) & \geq-2 T^{0}, \\
\left|V_{n}\right| & \leq C_{7},
\end{aligned}
$$

and

$$
\left.V_{n} \cdot \frac{\left(-X_{n}\right)}{\left|X_{n}\right|}\right|_{\left(\alpha_{n}(x, v), x, v\right)} \geq C_{3} .
$$

For $s \in\left[\max \left(\alpha_{n}, \alpha_{n+1}\right), 0\right]$ let

$$
d_{n}(s, x, v)=d_{n}(s)=\left|X_{n+1}(s)-X_{n}(s)\right|+\left|V_{n+1}(s)-V_{n}(s)\right| .
$$

Then

$$
\begin{aligned}
d_{n}(s)= & \left|\int_{s}^{0}\left(V_{n+1}-V_{n}\right) d u\right| \\
& +\left|\int_{s}^{0}\left(E_{n}\left(X_{n+1}\right)+V_{n+1} \wedge B\left(X_{n+1}\right)-E_{n-1}\left(X_{n}\right)-V_{n} \wedge B\left(X_{n}\right)\right) d u\right| \\
\leq & \int_{s}^{0}\left(d_{n}+\left\|\nabla E_{n}\right\|_{\infty} d_{n}+\left\|E_{n}-E_{n-1}\right\|_{\infty}+\|B\|_{\infty} d_{n}+\left|V_{n}\right|\|\nabla B\|_{\infty} d_{n}\right) d u \\
\leq & \int_{s}^{0}\left(C d_{n}+\left\|E_{n}-E_{n-1}\right\|_{\infty}\right) d u .
\end{aligned}
$$

Since

$$
s \geq \max \left(\alpha_{n}, \alpha_{n+1}\right) \geq \alpha_{n} \geq-2 T^{0}
$$

we have

$$
d_{n}(s) \leq 2 T^{0}\left\|E_{n}-E_{n-1}\right\|_{\infty}+C \int_{s}^{0} d_{n} d u
$$


and Gronwall's inequality yields

$$
\begin{aligned}
d_{n}(s) & \leq 2 T^{0}\left\|E_{n}-E_{n-1}\right\|_{\infty} e^{C|s|} \\
& \leq 2 T^{0}\left\|E_{n}-E_{n-1}\right\|_{\infty} e^{C 2 T^{0}}=C_{13}\left\|E_{n}-E_{n-1}\right\|_{\infty} .
\end{aligned}
$$

By (3.2) we have

$$
d_{n}(s)<C_{13} 2 C_{9} C_{7} \varepsilon
$$

We further restrict $\varepsilon$ so that

$$
C_{13} 2 C_{9} C_{7} \varepsilon<\frac{1}{2} C_{3}
$$

Now we choose $r$ by using Lemma 3.2 with $\delta=\frac{1}{2} C_{3}$. Further restrict $\varepsilon$ again so that

$$
C_{13} 2 C_{9} C_{7} \varepsilon<R-r
$$

Define

$$
D_{n}(x, v)=D_{n}=\sup \left\{d_{n}(s): s \in\left[\max \left(\alpha_{n}, \alpha_{n+1}\right), 0\right]\right\}
$$

and claim (with an additional restriction on $\varepsilon$ ) that

$$
\left|\alpha_{n+1}-\alpha_{n}\right| \leq 4 C_{3}^{-1} D_{n}
$$

and there is $C_{14}>0$ such that

$$
\left|f_{n+1}(x, v)-f_{n}(x, v)\right| \leq C_{14} \varepsilon D_{n} .
$$

To establish (3.12) consider the case that $\alpha_{n+1} \leq \alpha_{n}$ first. By (3.9) and (3.11) we have

$$
\begin{aligned}
\left|X_{n+1}\left(\alpha_{n}\right)\right| & \geq\left|X_{n}\left(\alpha_{n}\right)\right|-D_{n}=R-D_{n} \\
& \geq R-(R-r)=r .
\end{aligned}
$$

Also, by (3.9)

$$
\begin{aligned}
& \left.\left|V_{n+1} \cdot \frac{X_{n+1}}{\left|X_{n+1}\right|}-V_{n} \cdot \frac{X_{n}}{\left|X_{n}\right|}\right|\right|_{\alpha_{n}} \\
\leq & \left|V_{n+1}-V_{n}\right|+\left|V_{n}\right| \frac{\left|\left(X_{n+1}-X_{n}\right)\right| X_{n}\left|+X_{n}\left(\left|X_{n}\right|-\left|X_{n+1}\right|\right)\right|}{\left|X_{n+1}\right|\left|X_{n}\right|} \\
\leq & D_{n}+\frac{C_{7}}{C_{1}} 2 D_{n}<\left(1+\frac{2 C_{7}}{C_{1}}\right) C_{13} 2 C_{9} C_{7} \varepsilon .
\end{aligned}
$$

We restrict $\varepsilon$ so that

$$
\left(1+\frac{2 C_{7}}{C_{1}}\right) C_{13} 2 C_{9} C_{7} \varepsilon<\frac{1}{2} C_{3}
$$

Now using (3.7) we have

$$
\left.V_{n+1} \cdot \frac{\left(-X_{n+1}\right)}{\left|X_{n+1}\right|}\right|_{\alpha_{n}} \geq\left. V_{n} \cdot \frac{\left(-X_{n}\right)}{\left|X_{n}\right|}\right|_{\alpha_{n}}-\frac{1}{2} C_{3} \geq \frac{1}{2} C_{3} .
$$


Now by Lemma 3.2 we have

$$
\begin{aligned}
\alpha_{n+1} & \geq \alpha_{n}-\frac{R-\left|X_{n+1}\left(\alpha_{n}\right)\right|}{\frac{1}{2} \delta}=\alpha_{n}-\frac{\left|X_{n}\left(\alpha_{n}\right)\right|-\left|X_{n+1}\left(\alpha_{n}\right)\right|}{\frac{1}{4} C_{3}} \\
& \geq \alpha_{n}-\frac{4 D_{n}}{C_{3}},
\end{aligned}
$$

and (3.12) follows when $\alpha_{n+1} \leq \alpha_{n}$.

To establish (3.13) note first that by (3.9) we have

$$
D_{n} \leq C,
$$

so (3.12) implies

$$
\alpha_{n+1} \geq \alpha_{n}-4 C_{3}^{-1} D_{n} \geq-2 T^{0}-4 C_{3}^{-1} C=-C,
$$

so for all $s \in\left[\alpha_{n+1}, 0\right]$,

$$
\left|V_{n+1}(s)\right| \leq|v|+\|E\|_{\infty}|s| \leq C_{7}+C_{6} C=C .
$$

Hence,

$$
\begin{aligned}
& \left|f_{n+1}(x, v)-f_{n}(x, v)\right| \\
= & \varepsilon\left|F\left(X_{n+1}\left(\alpha_{n+1}\right), V_{n+1}\left(\alpha_{n+1}\right)\right)-F\left(X_{n}\left(\alpha_{n}\right), V_{n}\left(\alpha_{n}\right)\right)\right| \\
\leq & \varepsilon\|\nabla F\|_{\infty}\left(\left|X_{n+1}\left(\alpha_{n+1}\right)-X_{n}\left(\alpha_{n}\right)\right|+\left|V_{n+1}\left(\alpha_{n+1}\right)-V_{n}\left(\alpha_{n}\right)\right|\right) \\
\leq & C \varepsilon\left(\sup _{\left[\alpha_{n+1}, 0\right]}\left|V_{n+1}\right|\left|\alpha_{n+1}-\alpha_{n}\right|+\left|X_{n+1}\left(\alpha_{n}\right)-X_{n}\left(\alpha_{n}\right)\right|\right. \\
& \left.+C_{6}\left|\alpha_{n+1}-\alpha_{n}\right|+\left|V_{n+1}\left(\alpha_{n}\right)-V_{n}\left(\alpha_{n}\right)\right|\right) \\
\leq & C \varepsilon\left(\left|\alpha_{n+1}-\alpha_{n}\right|+D_{n}\right) \leq C \varepsilon D_{n},
\end{aligned}
$$

which is 3.13).

Establishing (3.12) and (3.13) in the case that $\alpha_{n+1}>\alpha_{n}$ is highly similar and is omitted. Also, (3.12) and (3.13) may be shown to hold in the case that $f_{n+1}(x, v) \neq 0$ with minor changes to the above estimates.

Combining (3.8) and (3.13) yields

$$
\left|f_{n+1}(x, v)-f_{n}(x, v)\right| \leq C \varepsilon\left\|E_{n}-E_{n-1}\right\|_{\infty}
$$

if $f_{n+1}(x, v) \neq 0$ or $f_{n}(x, v) \neq 0$. But if $f_{n+1}(x, v)=f_{n}(x, v)=0$, (3.14) still holds, so

$$
\left\|f_{n+1}-f_{n}\right\|_{\infty} \leq C \varepsilon\left\|E_{n}-E_{n-1}\right\|_{\infty} .
$$

But by Lemmas 2.3 and 2.5 we have

$$
\begin{aligned}
\left\|E_{n}-E_{n-1}\right\|_{\infty} & \leq C_{9}\left\|\rho_{n}-\rho_{n-1}\right\|_{\infty} \\
& \leq C_{9} \pi C_{7}^{2}\left\|f_{n}-f_{n-1}\right\|_{\infty}
\end{aligned}
$$


So

$$
\left\|f_{n+1}-f_{n}\right\|_{\infty} \leq C_{15} \varepsilon\left\|f_{n}-f_{n-1}\right\|_{\infty} .
$$

Requiring $\varepsilon<C_{15}^{-1}$ implies that $f_{n}$ converges uniformly on $\bar{D}$ to some continuous function $f$. Let

$$
\begin{gathered}
\rho=\int f d v \\
\left\{\begin{array}{c}
\Delta U=-4 \pi \rho \\
\left.U\right|_{\partial D}=0 \\
E=-\nabla U
\end{array}\right.
\end{gathered}
$$

Then $\rho_{n} \rightarrow \rho$ and $E_{n} \rightarrow E$ uniformly. By (3.8) and (3.12) it also follows that $\alpha_{n}$ converges uniformly; call the limit $\alpha$. From (3.8) it follows that $D_{n} \rightarrow 0$ uniformly, and hence $X_{n}$ and $V_{n}$ converge uniformly on $\left\{(s, x, v):(x, v) \in\left(D \times \mathbb{R}^{2}\right) \cup S_{+}\right.$and $\left.s \in(\alpha(x, v), 0]\right\}$, say to $X$ and $V$. Furthermore,

$$
\left\{\begin{array}{l}
X(s, x, v)=x+\int_{0}^{s} V(u, x, v) d u \\
V(s, x, v)=v+\int_{0}^{s}(E(X)+V \wedge B(X)) d u .
\end{array}\right.
$$

By Lemmas 3.1 and 2.4 we have

$$
\left\|\nabla f_{n}\right\|_{\infty} \leq C_{8} e^{C_{8}\left\|\nabla E_{n}\right\|_{\infty}} \varepsilon \leq C_{8} e^{C_{8} C_{11}} \varepsilon \leq C,
$$

so

$$
\begin{aligned}
|f(x, v)-f(y, w)| \leq & \left|f(x, v)-f_{n}(x, v)\right| \\
& +\left|f_{n}(x, v)-f_{n}(y, w)\right|+\left|f_{n}(y, w)-f(y, w)\right| \\
\leq & 2\left\|f_{n}-f\right\|_{\infty}+C|(x, v)-(y, w)|
\end{aligned}
$$

for all $n$. Hence

$$
|f(x, v)-f(y, w)| \leq C|(x, v)-(y, w)| .
$$

Also, $\rho$ is Lipschitz continuous, and it follows from Theorem 4.13 of [2] that $E$ is $C^{1}$. Now by (3.15) it follows that $X$ and $V$ are $C^{1}$. Then it further follows that $\alpha$ and hence

$$
f(x, v)=\varepsilon F(X(\alpha(x, v), x, v), V(\alpha(x, v), x, v))
$$

are $C^{1}$. Finally, that $f$ satisfies the Vlasov equation follows from (3.16), and the proof of Theorem 1.1 is complete.

Appendix.

Proof of Lemma 2.5. For $x \in D$ and $\varepsilon \geq 0$ let

$$
P^{\varepsilon}(x)=\int_{D} \rho(y) G^{\varepsilon}(x-y) d y
$$

where

$$
G^{\varepsilon}(z)=\ln \left(\sqrt{\varepsilon^{2}+|z|^{2}}\right)
$$


Let $\delta \in(0, R]$ and

$$
B_{\delta}=\left\{y \in \mathbb{R}^{2}:|x-y|<\delta\right\}
$$

Also let $e^{(1)}=(1,0)$ and $e^{(2)}=(0,1)$. Then for $\varepsilon>0$,

$$
\begin{aligned}
\partial_{x_{k} x_{\ell}} P^{\varepsilon}(x)= & \int_{D} \rho(y) \partial_{x_{k} x_{\ell}} G^{\varepsilon}(x-y) d y \\
= & \int_{D \backslash B_{\delta}} \rho(y) \partial_{x_{k} x_{\ell}} G^{\varepsilon}(x-y) d y \\
& -\int_{D \cap B_{\delta}} \rho(y) \nabla_{y} \cdot\left(\partial_{x_{\ell}} G^{\varepsilon}(x-y) e^{(k)}\right) d y \\
= & \int_{D \backslash B_{\delta}} \rho(y) \partial_{x_{k} x_{\ell}} G^{\varepsilon}(x-y) d y \\
& +\int_{D \cap B_{\delta}} \nabla \rho(y) \cdot e^{(k)} \partial_{x_{\ell}} G^{\varepsilon}(x-y) d y \\
& -\int_{\left(\partial B_{\delta}\right) \cap D} \rho(y) \partial_{x_{\ell}} G^{\varepsilon}(x-y) e^{(k)} \cdot n d S_{y} \\
& -\int_{(\partial D) \cap B_{\delta}} \rho(y) \partial_{x_{\ell}} G^{\varepsilon}(x-y) e^{(k)} \cdot n d S_{y} \\
= & I+I I+I I I+I V .
\end{aligned}
$$

Note that

$$
\left|\partial_{x_{k}} G^{\varepsilon}(z)\right| \leq \frac{1}{\sqrt{\varepsilon^{2}+|z|^{2}}}
$$

and

$$
\left|\partial_{x_{k} x_{\ell}} G^{\varepsilon}(z)\right| \leq \frac{3}{\varepsilon^{2}+|z|^{2}}
$$


so

$$
\begin{aligned}
|I| & \leq\|\rho\|_{\infty} \int_{D \backslash B_{\delta}} \frac{3}{\varepsilon^{2}+|x-y|^{2}} d y \\
& \leq 3\|\rho\|_{\infty} \int_{\delta}^{2 R} \frac{2 \pi r d r}{r^{2}}=6 \pi\|\rho\|_{\infty} \ln \left(\frac{2 R}{\delta}\right) \\
|I I| & \leq\|\nabla \rho\|_{\infty} \int_{D \cap B_{\delta}} \frac{d y}{\sqrt{\varepsilon^{2}+|x-y|^{2}}} \\
& \leq\|\nabla \rho\|_{\infty} \int_{0}^{\delta} \frac{2 \pi r d r}{r}=2 \pi\|\nabla \rho\|_{\infty} \delta \\
|I I I| & \leq\|\rho\|_{\infty} \int_{\left(\partial B_{\delta}\right) \cap D} \frac{1}{\sqrt{\varepsilon^{2}+|x-y|^{2}}} d S_{y} \\
& \leq\|\rho\|_{\infty} \int_{0}^{2 \pi} \frac{\delta d \theta}{\sqrt{\varepsilon^{2}+\delta^{2}} \leq 2 \pi\|\rho\|_{\infty}} .
\end{aligned}
$$

To estimate IV consider $x \neq 0$ and let

$$
n_{0}=\frac{x}{|x|}=\left(\cos \theta_{0}, \sin \theta_{0}\right) \text {. }
$$

Write

$$
\begin{aligned}
I V= & \int_{(\partial D) \cap B_{\delta}}\left((\rho(x)-\rho(y)) e^{(k)} \cdot n-\rho(x) e^{(k)} \cdot\left(n-n_{0}\right)\right. \\
& \left.-\rho(x) e^{(k)} \cdot n_{0}\right) \partial_{x_{\ell}} G^{\varepsilon}(x-y) d S_{y}=I V_{1}+I V_{2}+I V_{3} .
\end{aligned}
$$

Then

$$
\left|I V_{1}\right| \leq\|\nabla \rho\|_{\infty} \int_{(\partial D) \cap B_{\delta}}|x-y| \frac{d S_{y}}{\sqrt{\varepsilon^{2}+|x-y|^{2}}} \leq C\|\nabla \rho\|_{\infty} \delta .
$$

For $I V_{2}$ note that

$$
\begin{aligned}
|x-y|^{2} & =|x|^{2}+R^{2}-2 x \cdot y=|x|^{2}+R^{2}-2|x| R n_{0} \cdot n \\
& =|x|^{2}+R^{2}-|x| R\left(2-\left|n-n_{0}\right|^{2}\right) \\
& =(R-|x|)^{2}+|x| R\left|n-n_{0}\right|^{2},
\end{aligned}
$$

so for $|x| \leq \frac{1}{2} R$

$$
|x-y|^{2} \geq\left(R-\frac{1}{2} R\right)^{2}=\frac{1}{4} R^{2} \geq \frac{1}{16} R^{2}\left|n-n_{0}\right|^{2}
$$

and for $|x| \geq \frac{1}{2} R$,

$$
|x-y|^{2} \geq\left(\frac{1}{2} R\right) R\left|n-n_{0}\right|^{2}
$$


Hence

$$
\begin{aligned}
\left|I V_{2}\right| & \leq\|\rho\|_{\infty} \int_{(\partial D) \cap B_{\delta}} \frac{\left|n-n_{0}\right|}{\sqrt{\varepsilon^{2}+|x-y|^{2}}} d S_{y} \\
& \leq\|\rho\|_{\infty} \int_{\partial D} \frac{\left|n-n_{0}\right|}{\frac{1}{4} R\left|n-n_{0}\right|} d S_{y}=8 \pi\|\rho\|_{\infty} .
\end{aligned}
$$

For $\left|I V_{3}\right|$ note that

$$
I V_{3}=-\rho(x) e^{(k)} \cdot n_{0}\left(\int_{(\partial D) \cap B_{\delta}} \frac{x-y}{\varepsilon^{2}+|x-y|^{2}} d S_{y}\right)_{\ell} .
$$

It suffices to bound this integral when $x=(|x|, 0)$, in which case

$$
\int_{(\partial D) \cap B_{\delta}} \frac{x-y}{\varepsilon^{2}+|x-y|^{2}} d S_{y}=(\mathcal{I}, 0)
$$

where

$$
\mathcal{I}=\int_{(\partial D) \cap B_{\delta}} \frac{|x|-y_{1}}{\varepsilon^{2}+|x-y|^{2}} d S_{y}
$$

For $|x| \leq \frac{1}{2} R$

$$
\frac{|| x\left|-y_{1}\right|}{\varepsilon^{2}+|x-y|^{2}} \leq \frac{\frac{1}{2} R+R}{\left(\frac{1}{2} R\right)^{2}}=\frac{6}{R}
$$

so

$$
|\mathcal{I}| \leq \int_{\partial D} \frac{6}{R} d S_{y}=12 \pi
$$

Consider $|x| \geq \frac{1}{2} R$. Writing $y=R(\cos \theta, \sin \theta)$ with $|\theta| \leq \pi$ we have

$$
\begin{aligned}
\left|\frac{|x|-y_{1}}{\varepsilon^{2}+|x-y|^{2}}\right| & =\left|\frac{|x|-R \cos \theta}{\varepsilon^{2}+|x|^{2}+R^{2}-2|x| R \cos \theta}\right| \\
& =\frac{1}{2|x|}\left|1-\frac{\varepsilon^{2}+R^{2}-|x|^{2}}{\varepsilon^{2}+(R-|x|)^{2}+2|x| R(1-\cos \theta)}\right| \\
& \leq \frac{1}{2\left(\frac{1}{2} R\right)}\left(1+\frac{\varepsilon^{2}+R^{2}-|x|^{2}}{\varepsilon^{2}+(R-|x|)^{2}+2\left(\frac{1}{2} R\right) R C \theta^{2}}\right)
\end{aligned}
$$


and hence for $\varepsilon \leq 2 R$,

$$
\begin{aligned}
|\mathcal{I}| & \leq \int_{-\pi}^{\pi} R^{-1}\left(1+\frac{\varepsilon^{2}+R^{2}-|x|^{2}}{\varepsilon^{2}+(R-|x|)^{2}+C R^{2} \theta^{2}}\right) R d \theta \\
& \leq 2 \pi+\int_{-\infty}^{\infty} \frac{\varepsilon^{2}+R^{2}-|x|^{2}}{\varepsilon^{2}+(R-|x|)^{2}+C R^{2} \theta^{2}} d \theta \\
& =2 \pi+\left(\varepsilon^{2}+R^{2}-|x|^{2}\right) \frac{\pi}{\sqrt{C R^{2}} \sqrt{\varepsilon^{2}+(R-|x|)^{2}}} \\
& =2 \pi+C \frac{\varepsilon^{2}+R^{2}-|x|^{2}}{\sqrt{\varepsilon^{2}+(R-|x|)^{2}}} \leq 2 \pi+C \frac{\varepsilon^{2}+2 R(R-|x|)}{\frac{1}{\sqrt{2}}(\varepsilon+R-|x|)} \\
& =2 \pi+\sqrt{2} C \frac{2 R \varepsilon+2 R(R-|x|)}{\varepsilon+R-|x|}=C .
\end{aligned}
$$

Hence

$$
\left|I V_{3}\right| \leq C\|\rho\|_{\infty}
$$

and

$$
|I V| \leq C\|\rho\|_{\infty}+C\|\nabla \rho\|_{\infty} \delta
$$

for $x \neq 0$. By continuity (A.1) holds for $x=0$ also.

Collecting terms we have

$$
\left|\partial_{x_{k} x_{\ell}} P^{\varepsilon}(x)\right| \leq C\left(\|\rho\|_{\infty} \ln \left(\frac{2 R}{\delta}\right)+\|\nabla \rho\|_{\infty} \delta\right)
$$

for all $\delta \in(0, R]$. If $\|\rho\|_{\infty}<\|\nabla \rho\|_{\infty} R$ take

$$
\delta=\|\rho\|_{\infty} /\|\nabla \rho\|_{\infty},
$$

which yields

$$
\left|\partial_{x_{k} x_{\ell}} P^{\varepsilon}(x)\right| \leq C\|\rho\|_{\infty}\left(1+\ln \left(\frac{2 R\|\nabla \rho\|_{\infty}}{\|\rho\|_{\infty}}\right)\right) .
$$

If $\|\rho\|_{\infty} \geq\|\nabla \rho\|_{\infty} R$ take $\delta=R$, which yields

$$
\left|\partial_{x_{k} x_{\ell}} P^{\varepsilon}(x)\right| \leq C\|\rho\|_{\infty}+C R\|\nabla \rho\|_{\infty} \leq C\|\rho\|_{\infty} .
$$

In both cases it follows that

$$
\left|\partial_{x_{k} x_{\ell}} P^{\varepsilon}(x)\right| \leq C\left(1+\|\rho\|_{\infty}\right)\left(1+\ln \left(1+\|\nabla \rho\|_{\infty}\right)\right) .
$$

Note also that

$$
\begin{aligned}
\left|\partial_{x_{k}} P^{\varepsilon}(x)\right| & =\left|\int_{D} \rho(y) \partial_{x_{k}} G_{\varepsilon}(x-y) d y\right| \\
& \leq\|\rho\|_{\infty} \int_{D} \frac{d y}{\sqrt{\varepsilon^{2}+|x-y|^{2}}} \leq\|\rho\|_{\infty} \int_{0}^{2 R} \frac{2 \pi r d r}{r} \\
& \leq 4 \pi R\|\rho\|_{\infty} .
\end{aligned}
$$

Letting $\varepsilon \rightarrow 0$ shows that (A.2) and (A.3) hold for $\varepsilon=0$ also. 
Next let

$$
H(x)=\int_{D} \rho(y) \ln \left(\frac{|y|}{R}\left|x-\frac{R^{2} y}{|y|^{2}}\right|\right) d y
$$

for $x \in \bar{D}$. Then

$$
\left\{\begin{aligned}
\Delta\left(P^{0}-H\right) & =2 \pi \rho & & \text { in } D \\
P^{0}-H & =0 & & \text { on } \partial D .
\end{aligned}\right.
$$

Letting $z=R^{2} y /|y|^{2}$ we have $y=R^{2} z /|z|^{2}$ and

$$
d y=R^{4}|z|^{-4} d z,
$$

so

$$
H(x)=\int_{|z|>R} \rho\left(\frac{R^{2} z}{|z|^{2}}\right) \ln \left(\frac{R}{|z|}|x-z|\right) R^{4}|z|^{-4} d z .
$$

Let

$$
\sigma(z)=R^{4}|z|^{-4} \rho\left(\frac{R^{2} z}{|z|^{2}}\right)
$$

and

$$
C_{H}=\int_{|z|>R} \sigma(z) \ln \left(\frac{R}{|z|}\right) d z
$$

then

$$
H(x)=C_{H}+\int_{|z|>R} \sigma(z) \ln (|x-z|) d z .
$$

Note that

$$
\|\sigma\|_{\infty} \leq\|\rho\|_{\infty}
$$

and

$$
\begin{aligned}
\left|\partial_{z_{k}} \sigma(z)\right| & \leq R^{4}\left(4|z|^{-5}\|\rho\|_{\infty}+6 R^{2}|z|^{-6}\|\nabla \rho\|_{\infty}\right) \\
& \leq 4 R^{-1}\|\rho\|_{\infty}+6\|\nabla \rho\|_{\infty} .
\end{aligned}
$$

Proceeding as for $P^{0}$ yields

$$
\begin{aligned}
\left|\partial_{x_{k} x_{\ell}} H(x)\right| & \leq C\left(1+\|\sigma\|_{\infty}\right)\left(1+\ln \left(1+\|\nabla \sigma\|_{\infty}\right)\right) \\
& \leq C\left(1+\|\rho\|_{\infty}\right)\left(1+\ln \left(1+C\|\rho\|_{\infty}+C\|\nabla \rho\|_{\infty}\right)\right)
\end{aligned}
$$

and

$$
\left|\partial_{x_{k}} H(x)\right| \leq C\|\rho\|_{\infty} .
$$

Hence

$$
\left|\partial_{x_{k} x_{\ell}}\left(P^{0}-H\right)(x)\right| \leq C\left(1+\|\rho\|_{\infty}\right)\left(1+\ln \left(1+C\|\rho\|_{\infty}+C\|\nabla \rho\|_{\infty}\right)\right)
$$

and

$$
\left|\partial_{x_{k}}\left(P^{0}-H\right)(x)\right| \leq C\|\rho\|_{\infty}
$$

for $x \in D$. By Theorem 6.14 of [2], $P^{0}-H \in C^{2}(\bar{D})$, so (A.4) and (A.5) hold on $\bar{D}$. Lemma 2.5 now follows. 


\section{REFERENCES}

[1] J. Batt, W. Faltenbacher, and E. Horst, Stationary spherically symmetric models in stellar dynamics, Arch. Rational Mech. Anal. 93 (1986), no. 2, 159-183, DOI 10.1007/BF00279958. MR823117 (87i:85001)

[2] David Gilbarg and Neil S. Trudinger, Elliptic partial differential equations of second order, 2nd ed., Grundlehren der Mathematischen Wissenschaften [Fundamental Principles of Mathematical Sciences], vol. 224, Springer-Verlag, Berlin, 1983. MR737190(86c:35035)

[3] Robert T. Glassey, The Cauchy problem in kinetic theory, Society for Industrial and Applied Mathematics (SIAM), Philadelphia, PA, 1996. MR.1379589 (97i:82070)

[4] Yan Guo and Gerhard Rein, Isotropic steady states in galactic dynamics, Comm. Math. Phys. 219 (2001), no. 3, 607-629, DOI 10.1007/s002200100434. MR.1838751 (2002g:85001)

[5] Yan Guo, Singular solutions of the Vlasov-Maxwell system on a half line, Arch. Rational Mech. Anal. 131 (1995), no. 3, 241-304, DOI 10.1007/BF00382888. MR1354697 (96h:35228)

[6] Yan Guo, Regularity for the Vlasov equations in a half-space, Indiana Univ. Math. J. 43 (1994), no. 1, 255-320, DOI 10.1512/iumj.1994.43.43013. MR1275462 (95d:35178)

[7] Hyung Ju Hwang, Regularity for the Vlasov-Poisson system in a convex domain, SIAM J. Math. Anal. 36 (2004), no. 1, 121-171 (electronic), DOI 10.1137/S0036141003422278. MR2083855 (2005f:35036)

[8] P.-L. Lions and B. Perthame, Propagation of moments and regularity for the 3-dimensional VlasovPoisson system (English, with French summary), Invent. Math. 105 (1991), no. 2, 415-430, DOI 10.1007/BF01232273. MR.1115549 (92e:35160)

[9] K. Pfaffelmoser, Global classical solutions of the Vlasov-Poisson system in three dimensions for general initial data, J. Differential Equations 95 (1992), no. 2, 281-303, DOI 10.1016/00220396(92)90033-J. MR.1165424 (93d:35170)

[10] Gerhard Rein, Collisionless kinetic equations from astrophysics - the Vlasov-Poisson system, Handbook of differential equations: evolutionary equations. Vol. III, Handb. Differ. Equ., Elsevier/NorthHolland, Amsterdam, 2007, pp. 383-476, DOI 10.1016/S1874-5717(07)80008-9. MR2549372 (2011b:85005)

[11] Jack Schaeffer, Steady states for a one-dimensional model of the solar wind, Quart. Appl. Math. 59 (2001), no. 3, 507-528. MR.1848532(2002j:82114)

[12] Jack Schaeffer, Slow decay for a linearized model of the solar wind, Quart. Appl. Math. 70 (2012), no. 1, 181-198, DOI 10.1090/S0033-569X-2011-01252-2. MR2920623

[13] Jack Schaeffer, Steady states of the Vlasov-Maxwell system, Quart. Appl. Math. 63 (2005), no. 4, 619-643. MR 2187923 (2006k:82148)

[14] D. Tidman and N. Krall, Shock waves in collisionless plasmas, Wiley-Interscience, 1971. 